\title{
BALANCE HÍDRICO EN LA CUENCA ALTA DEL RíO REVENTAZÓN, COSTA RICA
}

\author{
Asdrúbal Vargas Sanabria \\ Escuela Centroamericana de Geología, Apdo. 35-2060 Universidad de Costa Rica \\ Correo electrónico: avargas @ cariari.ucr.ac.cr
}

(Recibido 21/10/1997; Aceptado 25/5/1998)

\begin{abstract}
A water balance was established for the upper part of the Reventazón river basin. The basin is controlled by 13 rain gauge stations. The rainy season ranges from May till November with an average precipitation of 3412 $\mathrm{mm} / \mathrm{y}$. The area of highest rain-fall is controlled by the station T-Seis.

The surface run-off is registered by the following stations: La Troya, Palomo, La Suiza and Angostura. The maximum surface run-off of about $300 \mathrm{~m}^{3} / \mathrm{s}$ was measured at the Angostura.station The minimum dry season surface run-off values are only $10 \%$ of this amount. The observation period ranges from 1977 to 1993 showing an annual average of 77 to $110 \mathrm{~m}^{3} / \mathrm{s}$. The average of the total period is $90 \mathrm{~m}^{3} / \mathrm{s}$.

Depending of the subcatchment area the rate of surface run-off to precipitation differs from $48 \%$ to $82 \%$ and concerning to this values the evapotranspiration varies from $18 \%$ to $52 \%$.

The groundwater discharge was determined by the method of Kille (1970) and Wundt (1953). The calculated values vary in the range of $15 \mathrm{l} / \mathrm{s} / \mathrm{km}^{2}$ at La Troya and $501 / \mathrm{s} / \mathrm{km}^{2}$ at Palomo.
\end{abstract}

\begin{abstract}
RESUMEN: En este trabajo se realizó un balance hídrico para la parte alta de la cuenca del río Reventazón. El análisis pluviométrico se ha llevado a cabo utilizando los datos de trece estaciones. La zona con mayor precipitación comprende a la estación T-Seis y la de menor precipitación encierra a la estación Sanatorio Durán. El promedio de precipitacion anual para el área de estudio fue estimado en $3412 \mathrm{~mm}$.

La cuenca está controlada por medio de las estaciones fluviométricas La Troya, Palomo, La Suiza y Angostura. En la estación Angostura los caudales mínimos diarios alcanzan solo el $10 \%$ de $105300 \mathrm{~m} / \mathrm{s}$ considerados como los valores máximos. Los valores promedio anuales durante el período $1977-1993$ se ubican entre 77 y 110 m³/s. El valor promedio anual durante dicho período se estimó en $90 \mathrm{~m} 3 / \mathrm{s}$. A partir del balance hídrico y dependiendo de la microcuenca se puede señalar que un $47-83 \%$ de la precipitación se considera como escorrentía total y un $17-53 \%$ como evapotranspiración.

El componente de la escorrentía total considerado como flujo base fue estimado utilizando los métodos de Kille (1970) y Wundt (1953). La recarga al acuífero alcanza para la región de la estación La Troya 15 1/s/km² y más de 50 1/s/ $/ \mathrm{km}^{2}$ para la microcuenca de la estación Palomo.
\end{abstract}

\section{INTRODUCCIÓN}

La cuenca del río Reventazón es una de las más grandes de Costa Rica y es uno de los sistemas hidrográficos más importantes debido al potencial hídrico que presenta. En ella se han construido el embalse El Llano, el cual abastece de agua a un sector del Área Metropolitana, el Proyecto Hidroeléctrico Cachí y en fase de construcción se encuentra el Proyecto Hidroeléctrico Angostura. Sin embargo, hasta el momento no se ha realizado un balance hídrico completo para 
medir el potencial hídrico de la cuenca, debido a que los datos se encuentran dispersos en diferentes instituciones.

Con este trabajo se pretenden compilar los datos hidrológicos necesarios para llevar a cabo un balance hídrico, el cual brinde una aproximación sobre el porcentaje de lluvia que implica el caudal de agua superficial, el caudal del flujo base y el valor de la evapotranspiración. Además, se espera contribuir a la explotación racional de los recursos hídricos de la cuenca.

El área de investigación se ubica en el centro de Costa Rica y abarca la cuenca alta del río Reventazón hasta el sitio del Proyecto Hidroeléctrico Angostura, el cual se construye cerca de la ciudad de Turrialba (Fig.1). La cuenca tiene un área de $1342 \mathrm{~km}^{2}$. Morfológicamente está muy diferenciada: en el área norte domina la Cordillera Volcánica Central con una pendiente moderada, la sección sur abarca territorios de pendiente alta de la Cordillera de Talamanca. La diferencia de altura entre el punto más bajo ubicado en el sitio denominado Angostura (543 m.s.n.m) y la elevación mayor en el Cerro de la Muerte (3491 m.s.n.m) alcanza los 3000 m.

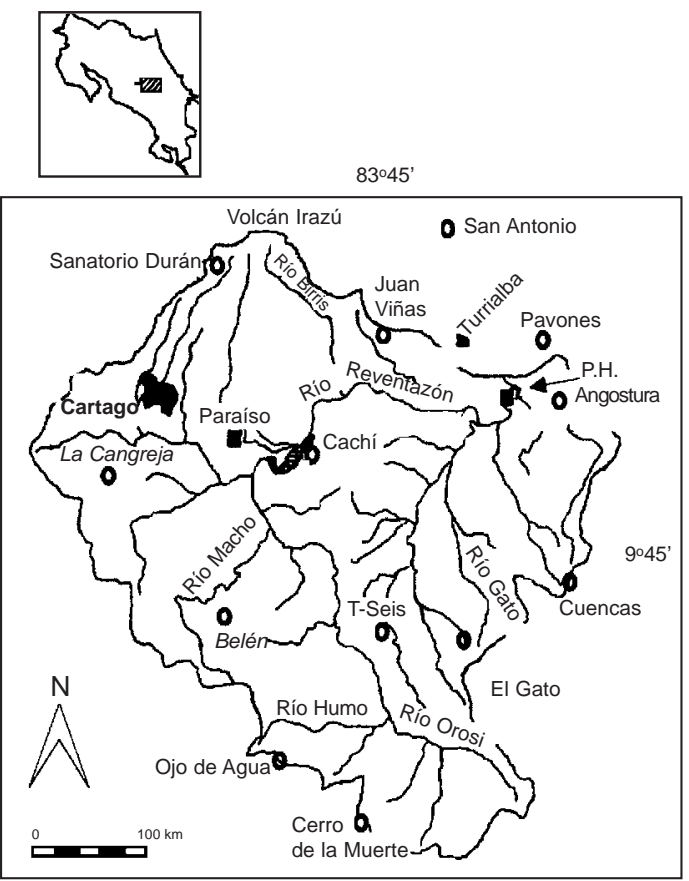

Fig. 1: Mapa de ubicación del área de estudio.

\section{PRECIPITACIÓN}

En el área de estudio se consideraron 13 estaciones pluviométricas con un registro diario de lluvia. Se analizaron los datos diarios durante los años 1979 y 1989 de las estaciones Sanatorio Durán, T-Seis, La Cangreja, La Suiza, Cachí y Cerro de la Muerte, las cuales se encuentran distribuidas en diferentes regiones dentro de la cuenca (Fig. 1). La figura 2 muestra la precipitación diaria para la estación Sanatorio Durán, la cual registra una precipitación relativa muy baja y la figura 3 presenta los valores para la estación T-Seis, en la cual se registra un nivel de precipitación relativo muy elevado. Los datos analizados demuestran que para la estación T- Seis no existe una verdadera estación seca; las restantes estaciones muestran un período seco mejor definido entre diciembre y marzo.
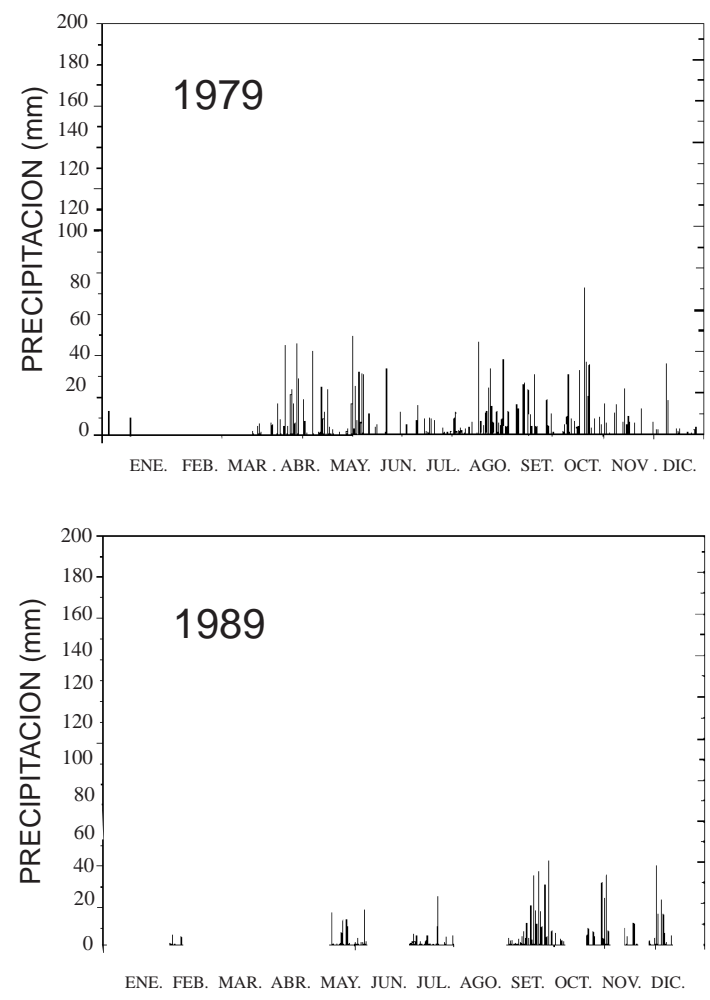

Fig. 2: Precipitación diaria, medida en los años 1979 y 1989 , en la estación Sanatorio Durán. 

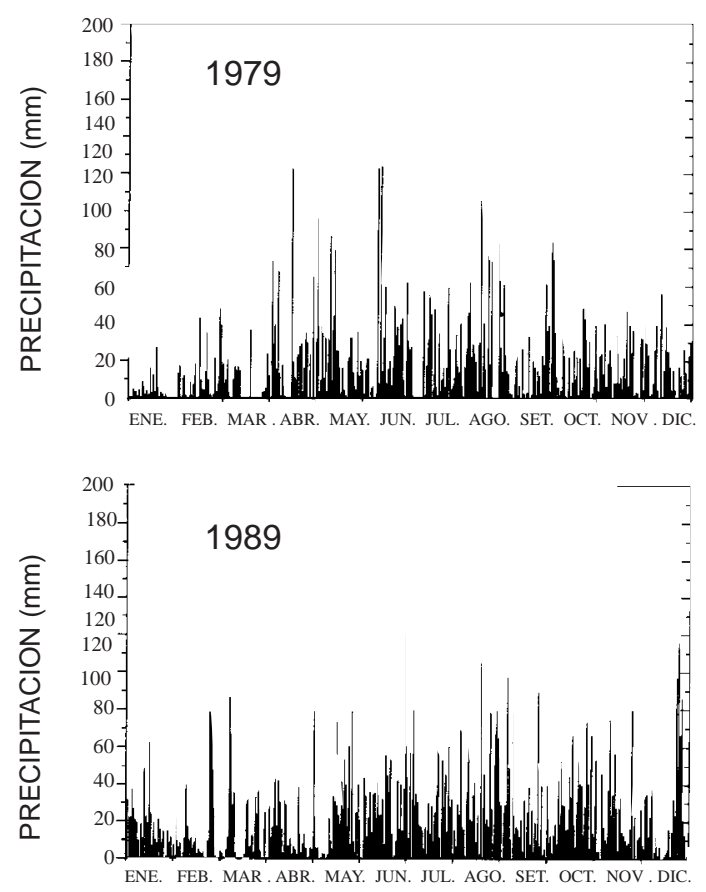

Fig. 3: Precipitación diaria, medida en los años 1979 y 1989 , en la estación T-Seis.

Las cantidades de lluvia diaria presentan variaciones muy amplias. En las estaciones Sanatorio Durán, Cerro de la Muerte, Cachí y la Suiza las lluvias alcanzan rara vez valores sobre los $60 \mathrm{~mm}$. En la estación T-Seis las lluvias diarias alcanzan valores superiores a los $80 \mathrm{~mm}$. Lo anterior demuestra que las cantidades de lluvia y su distribución son heterogéneas. Esto está además claramente mostrado por la evaluación de las cantidades de lluvia mensual y anual.

De los datos mensuales se estima que el mes más lluvioso es octubre. De las trece estaciones analizadas, 8 tienen el promedio mensual máximo en ese mes. El mes de setiembre ocupa el segundo puesto. El mes con menor precipitación es febrero (Fig. 4); en este mes 8 estaciones tienen su valor promedio mínimo para el período de estudio. El valor promedio máximo para el período 1977-1993 lo alcanza la estación T-Seis con $819 \mathrm{~mm}$. El valor promedio mínimo del mes

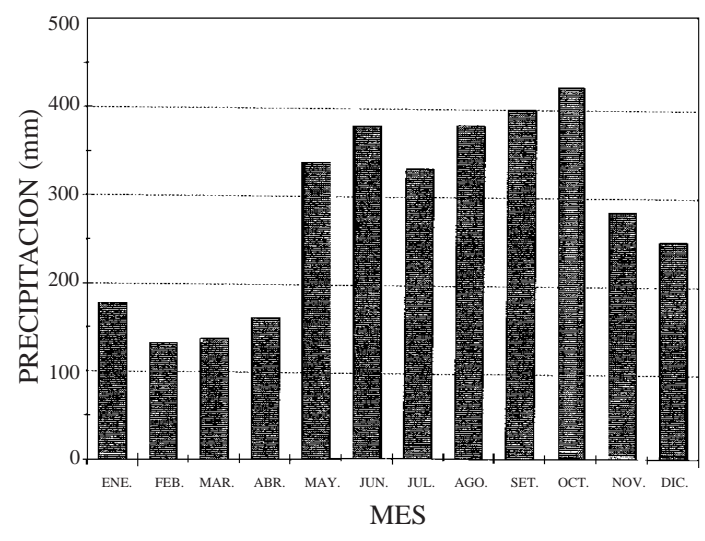

Fig. 4: Precipitación promedio mensual en el área de estudio.

de octubre ocurre en la estación Cachí con 198 $\mathrm{mm}$, la cual se ubica a solo $15 \mathrm{~km}$ de la estación T-Seis.

Con el análisis de la precipitación anual para el período de 1977-1993 se obtienen también marcadas diferencias. La estación T- Seis se ubica en el extremo superior con un valor promedio anual de $7492 \mathrm{~mm}$, seguida por las estaciones El Gato y Cuencas con $6693 \mathrm{~mm}$ y $4686 \mathrm{~mm}$, respectivamente. Geográficamente existe una banda territorial entre los 1600 y los 2000 m.s.n.m, la cual recibe abundantes precipitaciones, que se distribuyen durante todo el año. Las zonas muy elevadas o muy bajas reciben cantidades menores de lluvia. Con base en el promedio anual de precipitación de cada estación se obtiene una distribución de lluvia para el área de estudio (Fig. 5). Dicha ilustración permite, en asociación con la figura 1, establecer que el sistema de ríos secundarios del sector sur del área de estudio aporta una enorme cantidad de agua hacia el río Reventazón.

Considerando las grandes diferencias de lluvia en tan corta distancia, los valores promedio obtenidos deben ser tomados como aproximaciones. Por medio del método de Polígonos de Thiessen (1911) se obtuvo un valor promedio de $3412 \mathrm{~mm}$ para el área de estudio. 


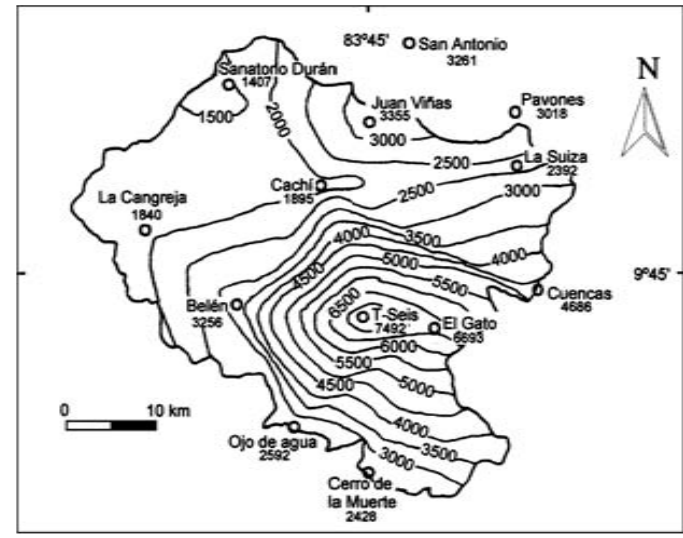

Fig. 5: Mapa de distribución de la precipitación.

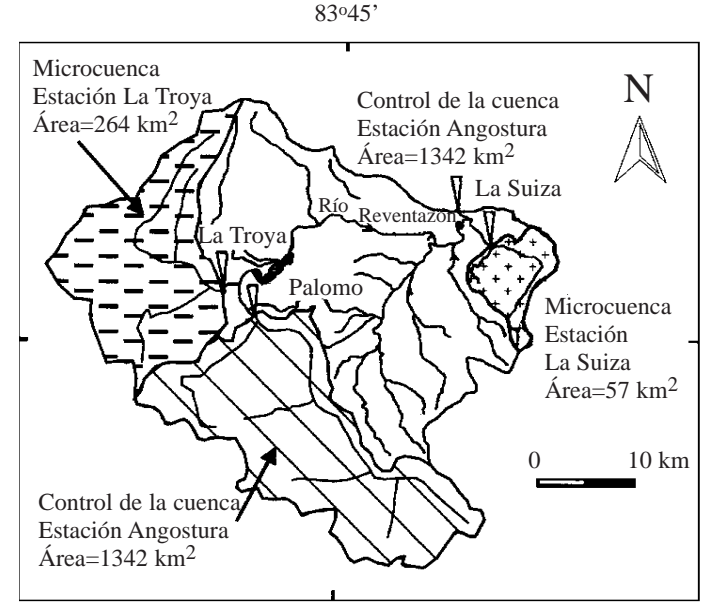

Fig. 6: Mapa de ubicación de las estaciones de medición de escorrentía y subcuencas.

\section{ANÁLISIS DE LOS DATOS DE LA ESTACIÓN ANGOSTURA}

Los datos de la estación fluviométrica Angostura documentan el caudal proveniente de la cuenca en su totalidad. El contorno de los hidrogramas permiten delimitar la época seca y húmeda para el período de 1990- 1992 (Fig. 7). Durante el período de verano, entre diciembre y abril, el caudal diario se reduce hasta valores por debajo de $\operatorname{los} 30 \mathrm{~m}^{3} / \mathrm{s}$. Los caudales máximos superan los $100 \mathrm{~m}^{3} / \mathrm{s}$. En la estación lluviosa se registran ocacionalmente valores de hasta $300 \mathrm{~m}^{3} / \mathrm{s}$ y los caudales mínimos superan $\operatorname{los} 50 \mathrm{~m} / \mathrm{s}$. Los caudales anuales promedio durante el período 1977-1993 varían entre 77 y $110 \mathrm{~m}^{3} / \mathrm{s}$ obteniéndose un valor promedio de $89,7 \mathrm{~m}^{3} / \mathrm{s}$. Existe una relativa gran diferencia entre los valores mínimos y máximos, mientras

Cuadro 1

Posición geográfica de las estaciones fluviométricas

\begin{tabular}{|c|c|c|c|c|c|}
\hline Estación & Río & Latitud & Longitud & Altura (m.s.n.m) & Datos mensuales anlizados \\
\hline Angostura & Reventazón & $9^{\circ} 53^{\prime}$ & $83^{\circ} 39^{\prime}$ & 583 & setiembre 1977-abril 1993 \\
\hline La Suiza & Tuis & $9^{\circ} 51$ & $83^{\circ} 37^{\prime}$ & 610 & diciembre1988-abril 1993 \\
\hline La Troya & Agua Caliente & $9^{\circ} 49^{\prime}$ & $83^{\circ} 52^{\prime}$ & 1029 & mayo 1981-abril 1993 \\
\hline Palomo & Grande de Orosí & $9^{\circ} 47^{\prime}$ & $83^{\circ} 50^{\prime}$ & 1077 & julio 1977-abril 1993 \\
\hline
\end{tabular}


que los valores promedio anuales muestran solamente oscilaciones moderadas (76,9-113,1

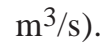

\section{ANÁLISIS DE LOS DATOS DE LAS ESTACIONES PALOMO, LA TROYA Y LA SUIZA}

En las estaciones Palomo, La Troya y la Suiza los caudales son mucho menores en comparación con los valores de la estación Angostura, debido al reducido tamaño de las cuencas. En los hidrogramas de las tres estaciones se observa la caida del caudal en diciembre y el aumento en mayo. Los caudales en estas estaciones durante la época lluviosa son aproximadamente de ocho veces más elevados que en la época seca.
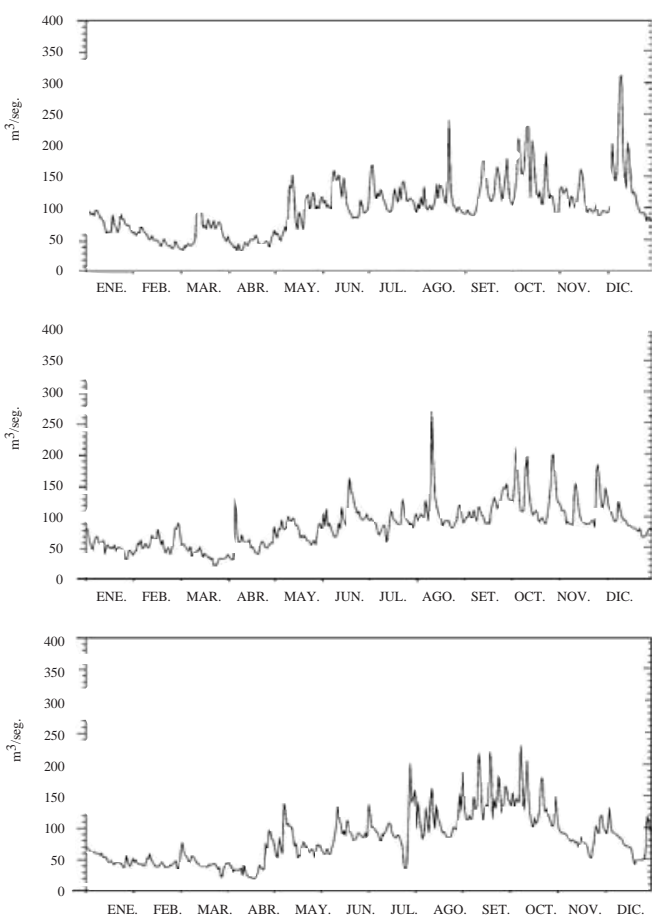

Fig. 7: Hidrograma del río Reventazón de los años 1990, 1991 y 1992 referido a la estación Angostura.
Para la estación Palomo, la cual controla la región con mayor cantidad de lluvia, se obtienen variaciones relativamente pequeñas de los caudales promedio. El caudal mínimo anual promedio se obtuvo para el año de 1992 con un valor de $27,8 \mathrm{~m}^{3} / \mathrm{s}$ y el máximo promedio en el año de 1981 con un valor de 41,7 m³/s. En comparación con la estación La Suiza y el sistema completo (Angostura), la Troya muestra amplias diferencias. El caudal mínimo promedio anual se registró en el año 1986 con un valor de $5,5 \mathrm{~m}^{3} / \mathrm{s}$ y el máximo promedio en el año 1981 con un valor de $11,4 \mathrm{~m}^{3} / \mathrm{s}$. Para la estación La Suiza, a pesar de contar con un registro de caudales reducido, se estimó el caudal promedio mínimo anual de $3,6 \mathrm{~m}^{3} / \mathrm{s}$ para el año de 1992 y el caudal promedio máximo anual de $5,1 \mathrm{~m}^{3} / \mathrm{s}$ para el año de 1993 . En conclusión las diferentes microcuencas se diferencian significativamente en el presupuesto de precipitaciones y caudales.

\section{COMPARACIÓN DE LOS DATOS}

Las estaciones comparables Angostura, Palomo, La Troya y La Suiza se diferencian principalmente en el tamaño de las microcuencas, en las características geológicas y en el presupuesto de las precipitaciones. El sistema fluvial del río Orosi pertenece a la microcuenca que controla la estación Palomo y es dicha microcuenca la que recibe la mayor cantidad de precipitaciones anuales. El comportamiento de la estación Angostura está en gran parte determinado por el sistema fluvial del río Orosi y por el régimen de lluvias en dicho territorio. El mayor aporte de escorrentía por área ocurre en la microcuenca de la estación Palomo (cuadro 2), mientras que el menor aporte sucede en el territorio controlado por la estación La Troya.

Las marcadas diferencias en el aporte areal de escorrentía son el resultado principalmente de las marcadas diferencias en la cantidad de precipitación, en el uso del suelo, en la geomorfología y en la geología de las microcuencas. 
Cuadro 2

Comparación de los valores de escorrentía

\begin{tabular}{lcccccc}
\hline Estación & Período & $\begin{array}{c}\text { Promedio anual } \\
\text { de escorrentía } \\
\left(\mathrm{m}^{3} / \mathrm{s}\right)\end{array}$ & $\begin{array}{c}\text { Área } \\
\left(\mathrm{km}^{2}\right)\end{array}$ & $\begin{array}{c}\text { Escorrentía por área } \\
\left(1 / \mathrm{s} / \mathrm{km}^{2}\right)\end{array}$ & $\begin{array}{c}\text { Caudal min. } \\
\left(\mathrm{m}^{3} / \mathrm{s}\right)\end{array}$ & $\begin{array}{c}\text { Caudal máx } \\
\left(\mathrm{m}^{3} / \mathrm{s}\right)\end{array}$ \\
\hline Angostura & $1977-1993$ & 89,7 & 1342,2 & 66,8 & 43,4 & 149,2 \\
Palomo & $1977-1993$ & 33,7 & 369,8 & 91,1 & 12,0 & 60,5 \\
La Troya & $1981-1993$ & 7,9 & 264,4 & 29,9 & 3,1 & 18,5 \\
La Suiza & $1988-1993$ & 4,4 & 56,8 & $77,5^{* *}$ & 2,2 & 7,0 \\
\hline
\end{tabular}

**Nov-dic. 1993 sin valores

\section{BALANCE HÍDRICO}

En el balance hídrico está comprendido la proporción entre la precipitación, la escorrentía y la evapotranspiración para el área de estudio en su totalidad y para las microcuencas antes señaladas. En primer lugar se cuantificaron las cantidades de precipitaciones y de escorrentía durante un período de años en particular para cada una de las regiones.

El cuadro 3 correspondiente al balance hídrico, muestra que la escorrentía para la estación Angostura para los años de estudio oscila entre el $57 \%$ y $69 \%$ de la precipitación. El caudal promedio de $89,7 \mathrm{~m}^{3} / \mathrm{s}$ (2108 $\mathrm{mm}$ ) calculado para el período de investigación 1977-1993 representa alrededor del $62 \%$ de la lluvia promedio. Para dicha estación se obtuvo además una correlación positiva entre la lluvia y la escorrentía (Fig. 8a), la cual permite establecer pronósticos de la reacción anual de la escorrentía sobre los acontecimientos anuales de la precipitación y con ello la relación entre la cantidad de lluvia anual y la reducción o aumento del volumen en el futuro embalse del Proyecto Hidroeléctrico Angostura.

La evapotranspiración se ha estimado como la diferencia entre la cantidad de lluvia y la escorrentía, la cual alcanza para la cuenca en general un $38 \%$ de la precipitación. El valor es relativamente menor a lo que se podría suponer y se puede explicar considerando que durante el
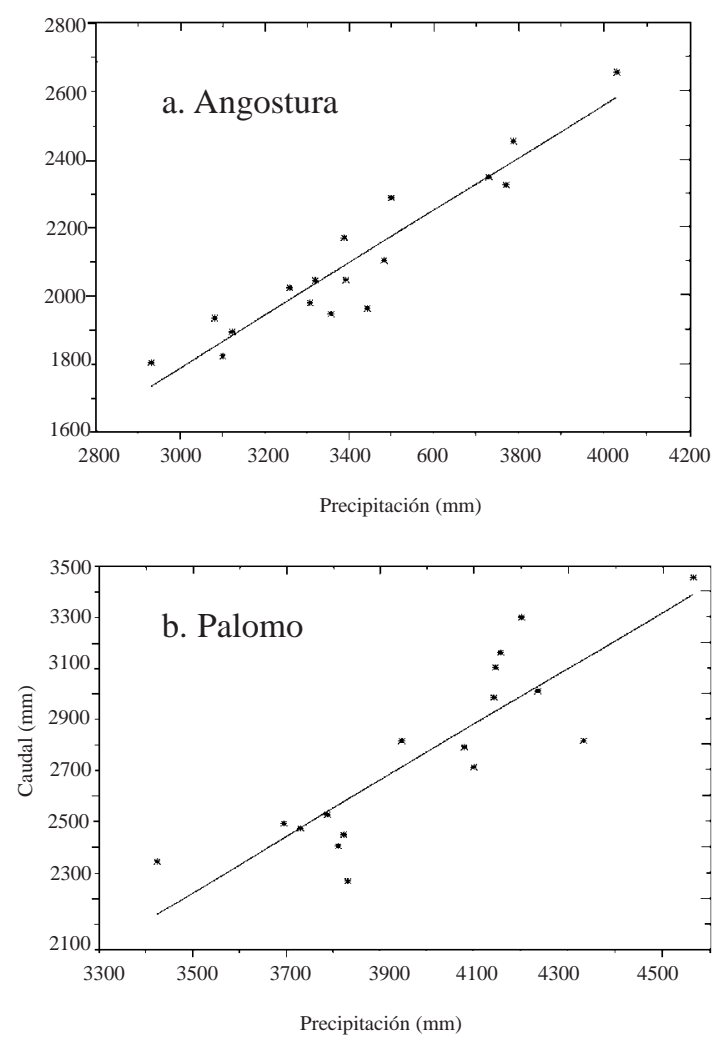

Fig. 8: Relación entre lluvia y caudal en las estaciones Angostura (a) y Palomo (b). 


\begin{tabular}{lccccc}
\hline $\begin{array}{l}\text { Estación } \\
\text { (período) }\end{array}$ & Lluvia (mm) & Escorrentía (mm) & \% de lluvia & $\begin{array}{c}\text { Evapotrans-piración } \\
(\mathrm{mm})\end{array}$ & \% de lluvia \\
$\begin{array}{l}\text { Angostura } \\
(1977-1993)\end{array}$ & 3412 & 2108 & 62 & 1304 & 38 \\
$\begin{array}{l}\text { Palomo } \\
(1977-1993)\end{array}$ & 4001 & 2875 & 72 & 1126 & 28 \\
$\begin{array}{l}\text { La Troya } \\
(1981-1993)\end{array}$ & 1973 & 940 & 48 & 1033 & 52 \\
$\begin{array}{l}\text { La Suiza } \\
(1988-1992)\end{array}$ & 2946 & 2427 & 82 & 519 & 18 \\
\hline
\end{tabular}

período lluvioso existe una humedad relativa casi del $100 \%$, lo cual reduce substancialmente la evapotranspiración. Además este proceso ocurre con mayor intensidad durante la época seca, donde, como ya se analizó, existen sectores donde ocacionalmente se presentan lluvias, las cuales sin embargo son reducidas en intensidad. Por otro lado la fuerte pendiente del terreno de algunos sectores de la cuenca estudiada favorece la escorrentía, reduce el tiempo de permanencia del agua y disminuye la evapotranspiración.

En el caso de la estación Palomo existe también una clara relación entre lluvia y escorrentía (Fig. 8b). Dicha estación mide la escorrentía proveniente en parte de la microcuenca del río Orosi, la cual recibe la mayor cantidad de lluvias. Dentro del área controlada por esta estación se ubica la estación T-Seis con una precipitación anual promedio de $7500 \mathrm{~mm}$. Además, en esta zona existe una amplia disribución de bosque nuboso, donde el aire se encuentra frecuentemente saturado con vapor de agua. La escorrentía correspondiente para los años 1977-1993 alcanza valores entre 62 y $81 \%$ de la precipitación. El promedio es de un $72 \%$, así que solo un $28 \%$ de la precipitación corresponde a la evapotranspiración (cuadro 3).

En los datos de la estación La Troya se aprecian condiciones diferentes. Dicha estación controla la escorrentía del sector Noroeste de la cuenca del río Reventazón, lo que corresponde a la falda sur del volcán Irazú. La precipitación promedio anual es de alrededor de $2000 \mathrm{~mm}$ y menos de la mitad de esta cantidad se asigna a la escorrentía. Para algunos años en particular donde las precipitaciones se encuentran muy por debajo del promedio anual de lluvia, la escorrentía no alcanza el $40 \%$.

Para la estación La Suiza se estimó un valor promedio de la escorrentía de alrededor del $82 \%$ de la precipitación. Esta es la estación con la escorrentía areal más alta y con tan solo un $18 \%$ de evapotranspiración. Efectivamente las precipitaciones alcanzan casi los $3000 \mathrm{~mm}$, la región contiene en gran parte bosque nuboso y presenta fuertes pendientes del terreno. En general se puede decir que existen marcadas diferencias en el balance hídrico de las microcuencas analizadas.

\section{FLUJO BASE}

Para realizar consideraciones sobre el flujo base se dispone de los datos de escorrentía medidos en las estaciones fluviométricas. Los hidrogramas muestran en el período seco (diciembre - abril) una caída o descenso. Sin embargo, este período seco no se caracteriza por una absoluta ausencia de lluvia, ya que ocacionalmente ocurren precipitaciones, las cuales no 
son de la misma intensidad que en el período de lluvias. Los hidrogramas muestran por lo tanto que la escorrentía no disminuye de manera regular; sin embargo evidencian que los valores son claramente menores que los de la estación lluviosa. Una disminución regular del caudal en el sentido de formar una curva de disminución se observa solo de manera restringida. Se puede partir, sin embargo, del hecho de que en una parte del sistema fluvial la escorrentía disminuye durante la estación seca, hasta estar formado exclusivamente por agua subterránea, es decir flujo base. Por otro lado se supone que durante la estación lluviosa el caudal disminuye ocacionalmente y está formado por agua subterránea.

En el análisis que se realizará a continuación se debe considerar, que en el área de estudio existe un fuerte relieve, lo cual facilita la escorrentía superficial. Además, a esto se suma la naturaleza de la precipitación, el nivel elevado de saturación del suelo y que este está en gran parte compuesto por materiales arcillosos relativamente poco permeables. En consecuencia la mayoría de las condiciones están dispuestas para favorecer una escorrentía superficial y evitar la infiltración del agua hacia niveles más profundos.

En este trabajo se utilizan los métodos estadísticos de Kille (1970) y Wundt (1953) para el cálculo del componente de agua subterránea en la escorrentía, los cuales por haber sido diseñados para condiciones diferentes a las del trópico deben tomarse con precaución. En el método de Wundt se estima el valor promedio de los caudales mínimos mensuales durante todo el año mientras que en el método de Kille se obtiene un valor que resulta de ordenar los valores mínimos mensuales en orden ascendente y obtener la mediana. Los valores obtenidos por ambos métodos se consideraron muy altos. Por lo tanto, además de los métodos anteriores, se estimaron los valores del flujo base por medio del método de Wundt utilizando solo los valores mínimos de la estación seca, los cuales se consideran como flujo de agua subterránea.

A partir de los datos de la estación La Troya se registran claras condiciones descendentes en el comportamiento de la escorrentía, ya que en el territorio controlado por esta estación, se presenta una estación seca bien definida. Aquí se presentan valores importantes de lluvia solo de manera ocacional. Esta es la única estación en la cual los datos al final del período húmedo permiten observar una curva de descenso en el hidrograma. El flujo base areal oscila entre $12 \mathrm{l} / \mathrm{s} / \mathrm{km}^{2}$ y $15 \mathrm{l} / \mathrm{s} / \mathrm{km}^{2}$ (cuadro 4). Con ello el flujo base presente en el caudal varía de un 39\% (Wundt en verano) hasta el $52 \%$ (Wundt), lo cual representa desde un $19 \%$ hasta un $25 \%$ del valor promedio de la precipitación anual.

También en la estación La Suiza se observa durante el período seco una curva regular de descenso. El hidrograma desciende después de varias inflexiones con una caida regular hasta los valores mínimos. Esto sucede durante una gran cantidad de meses, así que con los métodos establecidos se espera obtener una aproximación del valor del flujo base (cuadro 4). Como caudal de agua subterránea se obtiene un valor de alrededor de $2 \mathrm{~m}^{3} / \mathrm{s}$. Debido a que la microcuenca es de un tamaño reducido, se obtienen valores de flujo base de alrededor de $40 \mathrm{l} / \mathrm{s} / \mathrm{km}^{2}$, los cuales se consideran muy altos. Esto se puede explicar tomando en cuenta de que el área que controla esta estación recibe una cantidad de lluvia relativamente alta en comparación con el área de estudio en su totalidad y además porque el período de datos de caudales analizados es muy corto. El flujo base presente en la escorrentía varía de un 46\% (Wundt en verano) hasta el 57\% (Wundt), lo cual representa desde un $40 \%$ hasta un $49 \%$ del valor promedio de la precipitación anual.

En el caso de la estación Palomo, los valores mínimos de escorrentía medidos no permiten determinar con un grado deseable de precisión el flujo base. Durante la estación seca ocurren temporalmente inflexiones en el hidrograma, asociados a aumentos irregulares del caudal. Por lo tanto, los resultados se deben tomar con precaución. Es muy probable que los valores reales se encuentren por debajo de los valores estimados. Se debe además tomar en cuenta que la estación controla un territorio con un presupuesto de lluvia muy grande. Probablemente los valores reales corresponden a los valores estimados por Wundt para la estación seca. El flujo base estimado que se encuentra presente en la escorrentía varía de un $30 \%$ 
Cuadro 4

Flujo base en las microcuencas

\begin{tabular}{|c|c|c|c|}
\hline Parámetro & $\begin{array}{l}\text { Microcuenca } \\
\text { Palomo }\end{array}$ & $\begin{array}{c}\text { Microcuenca } \\
\text { La Troya }\end{array}$ & $\begin{array}{c}\text { Microcuenca } \\
\text { La Suiza }\end{array}$ \\
\hline Área $\left(\mathrm{km}^{2}\right)$ & 369,8 & 264,4 & 56,8 \\
\hline $\begin{array}{l}\text { Lluvia promedio } \\
(1977-1993 ; \mathrm{mm})\end{array}$ & 4001 & 1966 & 2816 \\
\hline Escorrentía promedio $\left(\mathrm{m}^{3} / \mathrm{s}\right)$ & 33,7 & 7,9 & 4,4 \\
\hline Flujo base Wundt (1/s/ km²) & 51 & 16 & 44 \\
\hline Porcentaje de lluvia & 40 & 25 & 49 \\
\hline Porcentaje de escorrentía & 56 & 52 & 57 \\
\hline Flujo base Killie (1/s/ km²) & 47 & 15 & 40 \\
\hline Porcentaje de lluvia & 37 & 24 & 44 \\
\hline Porcentaje de escorrentía & 52 & 50 & 51 \\
\hline $\begin{array}{l}\text { Flujo base Wundt } \\
\text { en verano }\left(1 / \mathrm{s} / \mathrm{km}^{2}\right)\end{array}$ & 27 & 12 & 36 \\
\hline Porcentaje de lluvia & 21 & 19 & 40 \\
\hline Porcentaje de escorrentía & 30 & 39 & 46 \\
\hline
\end{tabular}

(Wundt en verano) hasta el 56\% (Wundt), lo cual representa desde un $21 \%$ hasta un $40 \%$ del valor promedio de la precipitación anual.

Los datos de la estación Angostura muestran tanto en el período seco como durante la estación lluviosa un comportamiento muy irregular. No se puede apreciar una caída regular del hidrograma durante la estación seca, por el contrario ocacionalmente ocurren abruptos cambios. En general se tiene la impresión de que existe una distorción de los valores reales de escorrentía por causas antropogénicas, tales como la administración del Embalse de Cachí, las cuales modifican los datos y generan errores de una amplia magnitud, por lo que se ha excluido el análisis del flujo base para esta estación.

\section{CONCLUSIONES}

La distribución de la precipitación anual en la cuenca alta del río Reventazón no es uniforme y oscila entre poco menos de 1500 y más de 7000 $\mathrm{mm}$. Las estaciones T-Seis, Cuencas y el Gato se ubican en una de las regiones más lluviosas de Costa Rica y de América Latina. El valor promedio anual de las lluvias en la estación T-Seis supera los $7000 \mathrm{~mm}$. Las estaciones Sanatorio Durán, La Cangreja y Cachí se ubican en el sector de menor cantidad de lluvia dentro del área de estudio. Las regiones ubicadas a una elevación relativa muy baja así como aquellas ubicadas a una elevación muy alta reciben considerablemente menos precipitación que aquellas ubicadas a un nivel intermedio. Las lluvias están distribuidas de manera irregular durante el año. Según el método de Polígonos de Thiessen ( 1911) el valor promedio de la lluvia anual para el área de estudio alcanza $3412 \mathrm{~mm}$.

Con los datos de escorrentía de las estaciones fluviográficas Angostura, Palomo, La Troya y La Suiza se estimó una escorrentía areal promedio para las microcuencas que varía entre 30 y 90 1/s/ $/ \mathrm{km}^{2}$. Con ello de un $48 \%$ a un $80 \%$ de 
la precipitación del área de estudio se considera como escorrentía. Como flujo base se consideran valores entre 19 y $40 \%$ de la lluvia según el método de Wundt en verano.

Es necesario contar con una serie de datos de escorrentía más amplia para la estación La Suiza, con el fin de llevar a cabo un balance hídrico más preciso en el territorio controlado por dicha estación. Los datos de escorrentía para la estación Angostura no muestran un descenso regular después de la estación lluviosa, debido a procesos climáticos locales dentro de la cuenca y a factores antropogénicos, por lo cual la aplicación de los métodos de Wundt y Kille es muy limitada.

Las microcuencas analizadas muestran contrastes en los valores obtenidos de los balances hídricos producto de las diferencias en el tamaño, presupuesto de las precipitaciones, pendientes del terreno, uso de la tierra y la permeabilidad del suelo. La cuenca alta del río Reventazón presenta un potencial hídrico muy importante que debe ser protegido mediante prácticas adecuadas de uso del suelo.

\section{AGRADECIMIENTOS}

Se agradece la colaboración de Jorge Chávez de la Oficina de Geología y a los funcionarios del Departamento de Hidrología, del Instituto Costarricense de Electricidad por el apoyo brindado durante la realización de este trabajo.

\section{REFERENCIAS}

KILLE, K. 1970: Das Verfahren MoMNQ, ein Beitrag zur Berechnung der mittleren langjährigen Grundwasserneubildung mit Hilfe der monatlichen Niedrigabflüsse. Z. Deutsch.Geol. Ges., Sonderh. Hydrogeol. Hydrochem: 89-95.

THIESSEN, H., 1911: Precipitación for large areas. - Monthly Weather Rev., 39: 10821084.

WUNDT, W., 1953: Gewässerkunde. - 320 págs; Springer Verlag, Berlin. 